\title{
TITLE:
}

\section{Optical cross sections of deep levels in 4H-SiC}

$\operatorname{AUTHOR}(\mathrm{S}):$

Kato, M; Tanaka, S; Ichimura, M; Arai, E; Nakamura, S; Kimoto, T; Passler, R

\section{CITATION:}

Kato, M ... [et al]. Optical cross sections of deep levels in 4H-SiC. JOURNAL OF APPLIED PHYSICS 2006, 100(5): 053708.

ISSUE DATE:

2006-09-01

URL:

http://hdl.handle.net/2433/39720

\section{RIGHT:}

Copyright 2006 American Institute of Physics. This article may be downloaded for personal use only. Any other use requires prior permission of the author and the American Institute of Physics. 


\title{
Optical cross sections of deep levels in $4 \mathrm{H}$-SiC
}

\author{
M. Kato, ${ }^{\text {a) }}$ S. Tanaka, M. Ichimura, and E. Arai \\ Department of Engineering Physics, Electronics and Mechanics, Nagoya Institute of Technology, Gokiso, \\ Showa, Nagoya 466-8555, Japan \\ S. Nakamura and T. Kimoto \\ Department of Electronic Science and Engineering, Kyoto University, Kyotodaigaku-Katsura, Nishikyo, \\ Kyoto 615-8510, Japan \\ R. Pässler \\ Institut für Physik, Technische Universität Chemnitz, D-09107 Chemnitz, Germany
}

(Received 10 April 2006; accepted 19 June 2006; published online 13 September 2006)

\begin{abstract}
We have characterized deep levels in $4 \mathrm{H}$-SiC epilayers grown by cold wall chemical vapor deposition by the deep level transient spectroscopy (DLTS) and the optical-capacitance-transient spectroscopy (O-CTS). Four kinds of DLTS peaks were detected in the epilayers. Three of them are identified as the $Z_{1 / 2}, \mathrm{EH}_{6 / 7}$, and $\mathrm{RD}_{1 / 2}$ centers, while the other one has never been reported previously, and was named the NB center. On the basis of these DLTS data we have estimated the thermal ionization energies. The classical optical ionization energies of these centers, which are given by the sums of thermal ionization energies and Franck-Condon shifts, were estimated via fittings of the measured optical cross sections from O-CTS data by means of a sufficiently general theoretical model. Temperature dependences of nonradiative multiphonon carrier capture cross sections for the $Z_{1 / 2}$ and NB centers were roughly estimated in terms of parametrical dependences on thermal ionization energies and Franck-Condon shifts. (C) 2006 American Institute of Physics. [DOI: $10.1063 / 1.2344809$ ]
\end{abstract}

\section{INTRODUCTION}

In recent years, the crystalline quality of silicon carbide $(\mathrm{SiC})$ has been improved and its prospect for device application is expanding. Among the various $\mathrm{SiC}$ polytypes, $4 H$ $-\mathrm{SiC}$ is the most promising one for high power device applications because of its high breakdown voltage and high carrier mobility. In addition, high quality large size wafers are commercially available, ${ }^{1}$ and their epitaxial growth without introduction of a large amount of impurities is achieved by chemical vapor deposition (CVD). ${ }^{2,3}$ However, even in high quality $\mathrm{SiC}$ epilayers, microscopic defects, which generate deep levels in the band gap, still exist. Such deep levels affect the carrier dynamics, and their electrical and optical behaviors are thus important in view of device applications. In particular, the carrier lifetime, which is one of the most important parameters for bipolar devices, is as a rule significantly shortened due to the presence of deep levels. In fact it is normally very short (approximately microseconds) even in high quality $\mathrm{SiC}$ epilayers compared with that for $\mathrm{Si}^{2-4} \mathrm{In}$ order to lengthen the carrier lifetime, we have to characterize the influence of each type of deep levels on the carrier lifetime and then establish techniques for controlling the influence of the most detrimental deep levels. In addition, if carriers are captured into deep levels by the nonradiative multiphonon (NMP) emission mechanism, ${ }^{5,6}$ the energy emitted via the carrier capture process is converted into heat. This heat may enhance the defect formation or modify the

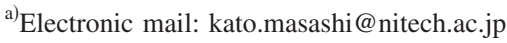

defect configuration around the original defects forming deep levels. Thus we should know in due detail the carrier dynamics related to deep levels.

In order to characterize deep levels in $4 H$-SiC, we employed in this study the deep level transient spectroscopy (DLTS) in combination with the optical-capacitance-transient spectroscopy (O-CTS) ${ }^{7-10}$ The O-CTS technique is convenient for measuring optical cross sections of deep levels. In particular, this technique is more sensitive and suitable for a distinction between different types of deep levels than other techniques that have been employed for estimations of optical cross sections of deep levels in SiC. ${ }^{11-13}$ By using DLTS and O-CTS here, we determine both the thermal activation energy and the energy spectrum of the cross section due to optical excitation of carriers from deep levels. Our numerical analyses of these optical spectra then provide estimates of the Franck-Condon shifts, which quantify the level-specific strength of the electron-phonon coupling. Finally, the approximate knowledge of thermal ionization energies and Franck-Condon shifts allows us to estimate possible temperature dependences of thermal electron capture cross sections that are due to the NMP capture mechanism.

\section{EXPERIMENT}

The samples used in this work were $n$-type $4 H$-SiC epilayers grown by horizontal cold wall CVD on $4 H-\mathrm{SiC}$ (0001) Si face substrates with $8^{\circ}$ inclination toward $\langle 11 \overline{2} 0\rangle$. The source gases were silane and propane, and the carrier gas was $\mathrm{H}_{2}$ for CVD growth. The gas flow rates for silane and $\mathrm{H}_{2}$ were fixed at $0.50 \mathrm{SCCM}$ (SCCM denotes cubic centimeter per minute at STP) and 3 SLM (standard liter per 


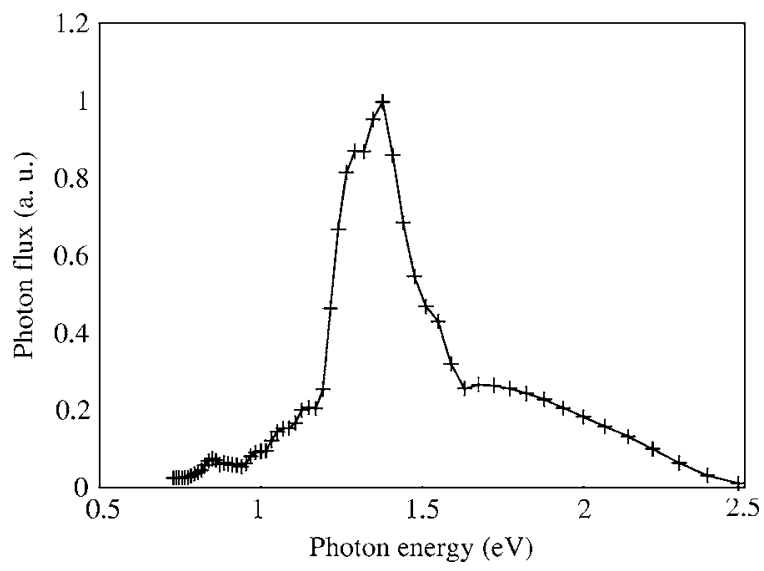

FIG. 1. Relative photon flux spectrum for Xe lamp used in O-CTS measurements.

minute), respectively, and two propane flow rates were chosen at 0.24 and $0.50 \mathrm{SCCM}$, which make a $\mathrm{C} / \mathrm{Si}$ ratio of 1.5 and 3.0. A part of deep level properties in these samples has been reported earlier. ${ }^{10,14} \mathrm{Au}$ or $\mathrm{Ni}$ was thermally evaporated on the epitaxial layer to form Schottky contacts. The Au contacts were thin enough to be transparent for excitation light, and the Schottky contact area was $1.77 \mathrm{~mm}^{2}$. We employed 0 and $-2 \mathrm{~V}$ for the filling pulse bias and the reverse bias, respectively, and the filling pulse width was $10 \mathrm{~ms}$ for both DLTS and O-CTS measurements. In the O-CTS measurements, we detected a capacitance-transient signal caused by the optical excitation of carriers from deep levels, and the signal was converted to an O-CTS spectrum by the rate window scan method. For the excitation light, a $300 \mathrm{~W}$ Xe lamp was employed. The light was passed through a monochromator and focused onto the Schottky contact region. The photon flux is of the order of $10^{16} \mathrm{~cm}^{-2} \mathrm{~s}^{-1}$, and the relative photon flux of the Xe lamp spectrum is shown in Fig. 1. For the O-CTS measurements, we employed the photon energy range of the excitation light of $1.02-2.38 \mathrm{eV}$. By the O-CTS measurements, we can estimate the optical cross section from $\sigma_{o}=1 /(\tau \Phi)$, where $\tau$ is the O-CTS peak time constant and $\Phi$ is the photon flux at given wavelength. (Note that since $\Phi$ is not constant in the measurement photon energy range, $\sigma_{o}$ does not linearly depend on the $1 / \tau$.) For the determination of the temperature dependence of electron capture cross sections, we have performed short filling pulse DLTS measurements by using a relay circuit and a function generator, which can produce a minimum pulse width of $50 \mathrm{~ns}$. For the short filling pulse DLTS measurements, we employed 0 and $-4 \mathrm{~V}$ for the filling pulse bias and the reverse bias, respectively.

\section{EXPERIMENTAL RESULTS}

\section{A. Deep levels in the $4 \mathrm{H}-\mathrm{SiC}$ epilayer grown at a $\mathrm{C} / \mathrm{Si}$ ratio of 1.5}

The net donor concentration in the sample grown at a $\mathrm{C} / \mathrm{Si}$ ratio of 1.5 was found to be $3.0 \times 10^{15} \mathrm{~cm}^{-3}$, and the DLTS spectrum for this sample with a time constant of $76 \mathrm{~ms}$ is shown in Fig. 1 of Ref. 10. Three peaks, $A, B$, and $C$, appear in the spectrum, and we obtain the Arrhenius plot

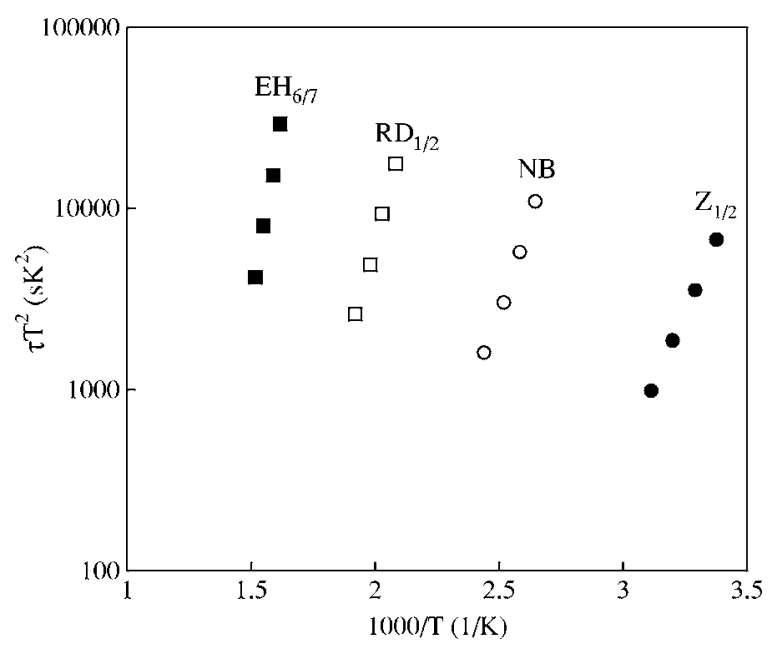

FIG. 2. Arrhenius plot for deep levels in $4 \mathrm{H}$-SiC epilayers observed by DLTS.

shown in Fig. 2. From this plot, peak $A$ has an activation energy $E_{A}$ of $0.61 \mathrm{eV}$ and a capture cross section at infinite temperature, $\sigma_{\infty}$, of $1.2 \times 10^{-15} \mathrm{~cm}^{2} .{ }^{10}$ These values are similar to the values reported for the $Z_{1 / 2}$ center. ${ }^{15,16}$ Thus, peak $A$ can be identified as the $Z_{1 / 2}$ center, and the trap concentration for peak $A$ is estimated from the peak height to be 3.0 $\times 10^{13} \mathrm{~cm}^{-3}$. O-CTS spectra due to the $Z_{1 / 2}$ center is shown in Fig. 2 of Ref. 10. The optical cross section $\sigma_{o}$ for the $Z_{1 / 2}$ center is obtained from the O-CTS spectra, as shown in Fig. 3 . To evaluate $\sigma_{o}$ at high photon energies at which the peak overlaps with another deeper level peak (peak $C$ ), we performed the same spectrum subtraction procedure as in our previous paper. ${ }^{8}$ It is generally accepted that the $Z_{1 / 2}$ center signal consists of two peaks, $Z_{1}$ and $Z_{2}$, as indicated by its subscript. ${ }^{13,15}$ However, we are not able to distinguish between $Z_{1}$ and $Z_{2}$ centers in our spectra. Therefore, throughout this paper, we treat peak $A$ as originating from a single center.

An estimation of $E_{A}$ and $\sigma_{\infty}$ for peak $B$ in Fig. 1 of Ref. 10 is difficult because of its small peak height. Thus we did not obtain these quantities from this figure. (Its concentration is estimated from the peak height to be of the order of $\left.10^{12} \mathrm{~cm}^{-3}\right)$. On the other hand, from Fig. 2, we obtain $E_{A}$ of

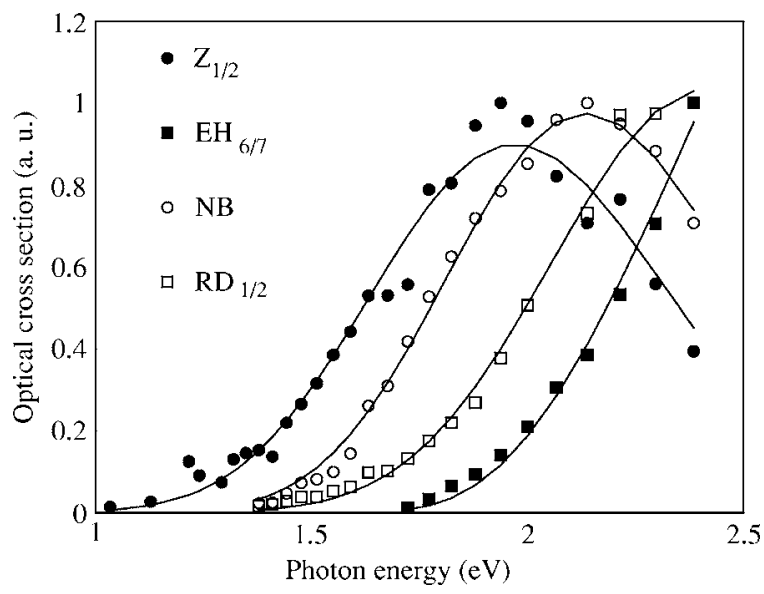

FIG. 3. Experimental data and fitting curves of the optical cross section. 


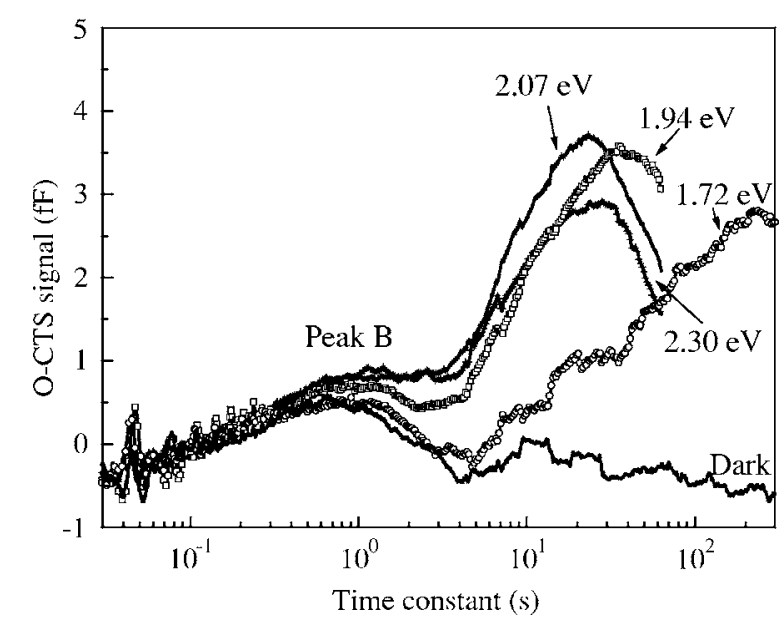

FIG. 4. O-CTS spectra measured at $350 \mathrm{~K}$ for the $4 H$-SiC epilayer grown at a $\mathrm{C} / \mathrm{Si}$ ratio of 1.5 .

$1.65 \mathrm{eV}$ and $\sigma_{\infty}$ of $3.0 \times 10^{-13} \mathrm{~cm}^{2}$ for peak $C$, and the trap concentration for peak $C$ is estimated to be $2.0 \times 10^{13} \mathrm{~cm}^{-3}$. Since our $E_{A}$ and $\sigma_{\infty}$ values for peak $C$ are almost the same as those reported for the $\mathrm{EH}_{6 / 7}$ center, ${ }^{4,17}$ peak $C$ can be ascribed to the $\mathrm{EH}_{6 / 7}$ center. Figure 4 shows the O-CTS spectra measured at $350 \mathrm{~K}$, in all of which a small peak originating from thermal excitation of peak $B$ appears around $0.7 \mathrm{~s}$. Without illumination, no peak is observed other than peak $B$, while with $1.72 \mathrm{eV}$ illumination, another peak arises around $200 \mathrm{~s}$. With more than $1.72 \mathrm{eV}$ illumination energy, this peak appears within a time constant interval of 10-100 s. Since this peak has similar peak heights $(\sim 3 \mathrm{fF})$ as the $\mathrm{EH}_{6 / 7}$ center peak in Fig. 1 of Ref. 10, we can conclude that this peak originates from optical excitation of the $\mathrm{EH}_{6 / 7}$ center. We derive $\sigma_{o}$ for the $\mathrm{EH}_{6 / 7}$ center, as shown in Fig. 3. The peak for the $\mathrm{EH}_{6 / 7}$ center is also considered to actually consist of two overlapping peaks, $\mathrm{EH}_{6}$ and $\mathrm{EH}_{7},{ }^{17}$ but we do not see any separation of the peak. Thus, we treat the peak as originating from a single center.

\section{B. Deep levels in the $4 \mathrm{H}-\mathrm{SiC}$ epilayer grown at a $\mathrm{C} / \mathrm{Si}$ ratio of 3.0}

The sample grown at a $\mathrm{C} / \mathrm{Si}$ ratio of 3.0 has a net donor concentration of $4.1 \times 10^{14} \mathrm{~cm}^{-3}$. The DLTS spectrum with a time constant of $76 \mathrm{~ms}$ is shown in Fig. 5. Two peaks are observed around 380 and $470 \mathrm{~K}$, and the peak at $380 \mathrm{~K}$ seems to be the same as peak $B$ in Fig. 1 in Ref. 10 because of the similarity in the peak position. Thus, we denote the peak at $380 \mathrm{~K}$ as peak $B$ and the peak at $470 \mathrm{~K}$ as peak $D$. $E_{A}$ and $\sigma_{\infty}$ for peak $B$ obtained from the Arrhenius plot are $0.80 \mathrm{eV}$ and $1.5 \times 10^{-15} \mathrm{~cm}^{2}$, respectively, and the trap concentration is estimated as $1.8 \times 10^{12} \mathrm{~cm}^{-3}$. Although this concentration is almost the same as that of peak $B$ in the sample grown at a $\mathrm{C} / \mathrm{Si}$ ratio of 1.5 , peak $B$ appears more clearly in Fig. 5 because of the lower net donor concentration. Hereafter, we label the trap introducing peak $B$ as the NB center. The O-CTS spectra measured at $150 \mathrm{~K}$ are shown in Fig. 6. With $1.51 \mathrm{eV}$ illumination, a peak appears around $30 \mathrm{~s}$, and its peak height is similar to the DLTS peak height for the NB center $(\sim 4 \mathrm{fF})$. Thus we consider this O-CTS peak to origi-

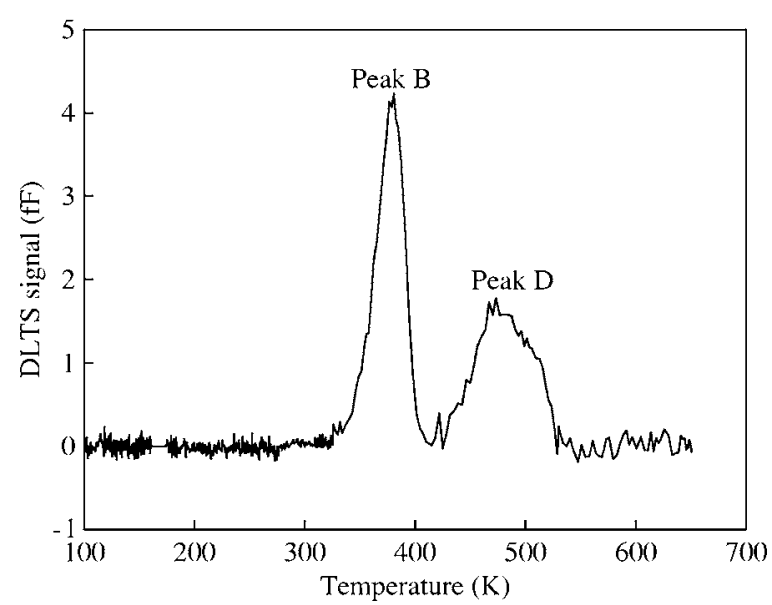

FIG. 5. DLTS spectrum for the $4 H$-SiC epilayer grown at a $\mathrm{C} / \mathrm{Si}$ ratio of 3.0 $(\tau=76 \mathrm{~ms})$.

nate from the optical excitation of the NB center. The time constant of the peak changes and the peak height increases with increasing illumination energy. This peak height increase is due to an overlap of peak $B$ with a peak originating from another deeper level (peak $D$ ). Since the height of peak $D$ is small compared with that of peak $B$, we ignore the influence of peak $D$ for our evaluation of the time constant of peak $B$. From the O-CTS spectra we obtain the photon energy dependence of $\sigma_{o}$ for the NB center, as shown in Fig. 3.

For peak $D, E_{A}$ and $\sigma_{\infty}$ obtained from the Arrhenius plot (Fig. 2) are $1.01 \mathrm{eV}$ and $8.4 \times 10^{-16} \mathrm{~cm}^{2}$, respectively, and the trap concentration is $7.6 \times 10^{11} \mathrm{~cm}^{-3}$. We can ascribe peak $D$ to the $\mathrm{RD}_{1 / 2}$ center because the estimated $E_{A}$ and $\sigma_{\infty}$ values are similar to those previously reported for the $\mathrm{RD}_{1 / 2}$ center. ${ }^{16}$ We show in Fig. 7 several O-CTS spectra measured at $370 \mathrm{~K}$, and all these spectra show a peak around $0.2 \mathrm{~s}$ irrespective of the illumination energies. This peak is ascribed to thermal excitation of electrons from the NB center because the time constant nearly corresponds to that for the thermal electron emission. Without illumination, a shoulder of the peak originating from thermal excitation of the $\mathrm{RD}_{1 / 2}$ center is observed in the spectrum. The peak position changes depending on the illumination energies. From these O-CTS spectra, we obtain $\sigma_{o}$ for the $\mathrm{RD}_{1 / 2}$ center, as shown

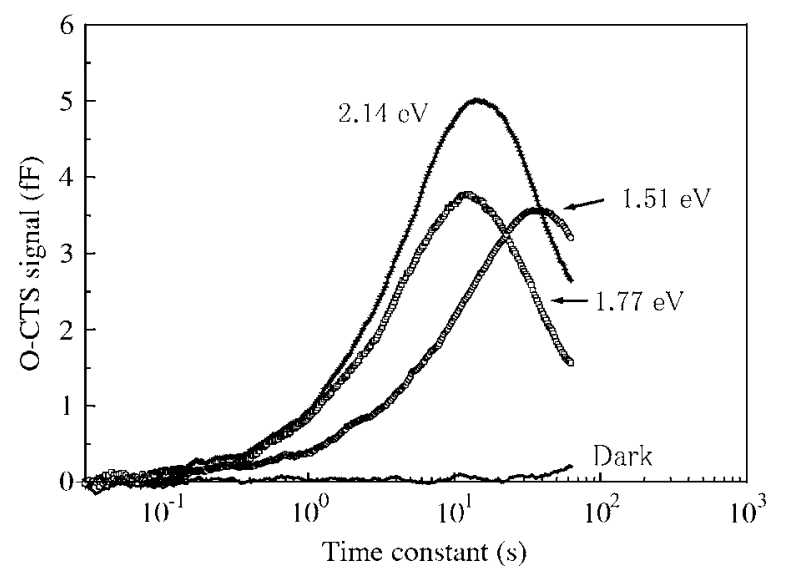

FIG. 6. O-CTS spectra measured at $150 \mathrm{~K}$ for the $4 H$-SiC epilayer grown at a $\mathrm{C} / \mathrm{Si}$ ratio of 3.0 . 


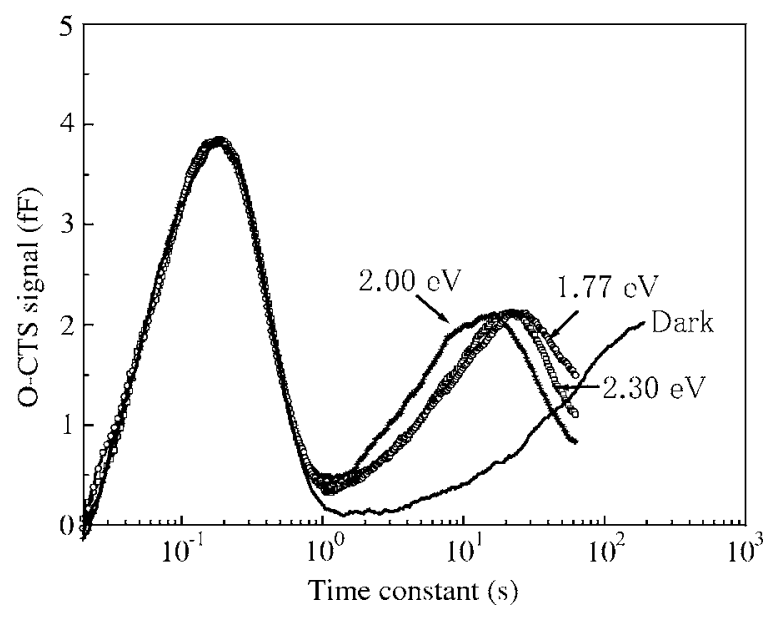

FIG. 7. O-CTS spectra measured at $370 \mathrm{~K}$ for the $4 \mathrm{H}$-SiC epilayer grown at a $\mathrm{C} / \mathrm{Si}$ ratio of 3.0.

in Fig. 3. (The peak for $2.30 \mathrm{eV}$ illumination has a larger peak time constant than the peak for $2.00 \mathrm{eV}$ illumination because the photon flux is smaller at $2.30 \mathrm{eV}$ than at $2.0 \mathrm{eV}$, as shown in Fig. 1.)

We performed the short filling pulse DLTS measurements for this sample because temperature dependences of the electron capture cross sections for the $\mathrm{NB}$ and $\mathrm{RD}_{1 / 2}$ centers have never been reported. The DLTS spectra with filling pulses of $10 \mathrm{~ms}, 1 \mu \mathrm{s}$, and $50 \mathrm{~ns}$ are shown in Fig. 8 (with the time constant of $332 \mathrm{~ms}$ ). Although the peak height for the NB center is slightly reduced with 50 ns filling pulse, the reduction of the peak height is very small. Therefore, the capture time constant should be less than $50 \mathrm{~ns}$. Thus the corresponding capture cross section at the peak temperature for the NB center should be larger than $2.2 \times 10^{-15} \mathrm{~cm}^{2}$. Since the capture cross section at infinite temperature for the NB center is about $1.5 \times 10^{-15} \mathrm{~cm}^{2}$, the temperature dependence of the capture cross section for the NB center is expected to be very small. On the other hand, the height and shape of the $\mathrm{RD}_{1 / 2}$ center peak change markedly with filling pulse width. The change of the shape indicates that the peak for the $\mathrm{RD}_{1 / 2}$ center consists of several overlapping peaks, as suggested in previous reports. ${ }^{16}$ Thus it is difficult to draw

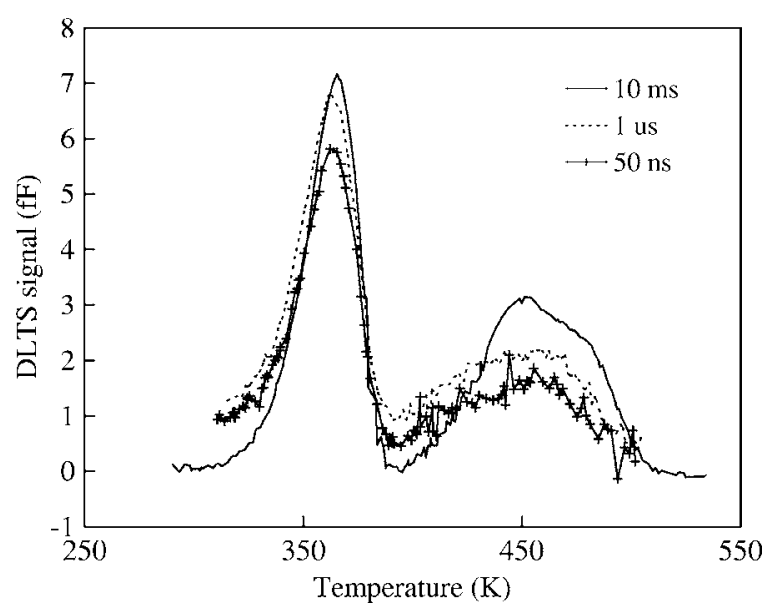

FIG. 8. The DLTS spectra with filling pulses of $10 \mathrm{~ms}, 1 \mu \mathrm{s}$, and $50 \mathrm{~ns}$ for the $4 \mathrm{H}$-SiC epilayer grown at a $\mathrm{C} / \mathrm{Si}$ ratio of 3.0. any conclusion concerning the temperature dependence of the capture cross section for the $\mathrm{RD}_{1 / 2}$ center.

We also performed series of the DLTS measurements at different magnitudes of reverse biases, without finding any electric field dependence of the thermal activation energies $E_{A}$. This indicates that the centers under study are effectively neutral in the processes of electron emission, which implies that the corresponding deep levels are acceptorlike. Thus the associated optical excitation processes (measured here by O-CTS) are not affected ${ }^{18}$ by an additional attractive or repulsive Coulombic potential, ${ }^{19}$ and there is no need here to include a corresponding Sommerfeld factor ${ }^{19}$ into the theoretical model (see below) for numerical analyses of measured optical cross sections.

\section{THEORETICAL FITTING AND DISCUSSION}

Optical cross sections of deep levels can be generally represented by convolution integrals of the form ${ }^{20,21}$

$$
\sigma_{o}(h \nu, T)=\frac{1}{h \nu} \int d E_{e} \sigma_{e}\left(E_{e}\right) E_{e} R\left(h \nu-E_{e}, T\right),
$$

where the $T$-independent electronic factor, $\sigma_{e}\left(E_{e}\right)$, represents the cross sections for absorption of variable (final-statespecific) electronic energy portions, $E_{e}$, by the excited electron (i.e., without phonon participation). The associated $T$-dependent Franck-Condon factor $R\left(h \nu-E_{e}, T\right)$ quantifies the probabilities of changing into heat of the differences between the energy of the absorbed photon $h \nu$, and the individual electronic excitation energies $E_{e}$ by means of corresponding multiphonon emission or absorption processes, respectively. In this paper, we adopt a Taylor series expansion form given by Eq. (A4) in Ref. 21 for $\sigma_{e}\left(E_{e}\right)$ and a sufficiently elaborate form given by Eq. (A14) in Ref. 20 for $R\left(h \nu-E_{e}, T\right)$. Thus Eq. (1) can be reduced to the approximate form

$$
\begin{aligned}
\sigma_{o}(h \nu, T) \propto & \frac{1}{h \nu} \int_{0}^{(+\infty)} d E_{k} \frac{1}{\sqrt{2 \pi \varepsilon F\left(h \nu-E_{k}-E^{T}, T\right)}} \\
& \times \sqrt{E_{k}}\left(1+\sum_{n=1}^{\geqslant 2} c_{n} E_{k}{ }^{n}\right) \\
& \times \exp \left[\frac{F\left(h \nu-E_{k}-E^{T}, T\right)-F(T)}{\varepsilon}\right. \\
& -\frac{h \nu-E_{k}-E^{T}}{\varepsilon} \\
& \left.\times \ln \frac{h \nu-E_{k}-E^{T}+F\left(h \nu-E_{k}-E^{T}, T\right)}{D+F(T)}\right],
\end{aligned}
$$

where $D$ is the Franck-Condon (FC) shift, $\varepsilon$ is the effective (average) phonon energy, $E^{T}$ is the thermal ionization energy (=zero-phonon absorption threshold), $E_{k} \equiv E_{e}-E^{T}$ is the kinetic energy of the excited carrier, and the functions $F(h \nu$ $\left.-E_{k}-E^{T}, T\right)$ and $F(T)$ have been defined as Eqs. (A15) and (A16) in Ref. 20. Within this representation, the thermally broadened optical cross sections are given in terms of parametrical dependences on several level-specific fitting parameters, namely, the thermal ionization (zero-phonon threshold) 
TABLE I. Parameter values mediating our fittings of measured optical cross section curves, for the four deep levels under study, by means of Eq. (2). Included are the corresponding magnitudes for optical ionization energies, $E^{O} \equiv E^{T}+D$.

\begin{tabular}{cccccc}
\hline \hline Center & $E^{T}(\mathrm{eV})$ & $D(\mathrm{eV})$ & $E^{O}(\mathrm{eV})$ & $c_{1}\left(\mathrm{eV}^{-1}\right)$ & $c_{2}$ (fited) $\left(\mathrm{eV}^{-2}\right)$ \\
\hline$Z_{1 / 2}$ & 0.55 & 1.32 & 1.87 & 1.3 & -10 \\
$\mathrm{EH}_{6 / 7}$ & 1.65 & 0.30 & 1.95 & 10.8 & 0 \\
$\mathrm{NB}$ & 0.80 & 1.28 & 2.08 & 0.1 & -10 \\
$\mathrm{RD}_{1 / 2}$ & 1.01 & 1.24 & 2.25 & 2.9 & -10 \\
\hline \hline
\end{tabular}

energy $E^{T}$, the Franck-Condon (FC) shift $D$, the effective phonon energy $\varepsilon$, and at least two expansion coefficients $c_{1}$ and $c_{2}$. The associated classical optical ionization energy is known to be given just by the sum, ${ }^{20} E^{O} \equiv E^{T}+D$, of the thermal ionization energy and the the FC shift.

The optical data, which are presently available for various deep levels in $4 H-\mathrm{SiC}$, are not sufficient for an unambiguous determination of all these (i.e., at least five) empirical trap parameters occurring in Eq. (2). Yet, we can reduce the existing uncertainties by adopting plausible fixed values for these parameters. Firstly, assuming that significant contributions to the thermal broadening of the deep level optical cross sections in $\mathrm{SiC}$ are made only by lattice modes, we can a priori limit the probable range for $\varepsilon$ to about $0.06-0.10 \mathrm{eV}^{21}$ In this paper, we adopt an intermediate value of $0.08 \mathrm{eV}$ for $\varepsilon$. Secondly, for $E^{T}$ we have used activation energies obtained from DLTS measurements as fixed parameters in Eq. (2). Thirdly, the high-energy wings of measured $\sigma_{o}(h \nu, T)$ spectra are somewhat too short for an unambiguous, simultaneous determination of the electronic expansion coefficients $c_{1}$ and $c_{2}$. In view of the limitation of our experimental data sets we have fixed the second expansion coefficients at values of either 0 or $-10 \mathrm{eV}^{-2}$ (Table I), which turned out to be compatible with the behavior of the upper parts of measured $\sigma_{o}(h \nu, T)$ spectra. Consequently, owing to the obvious (from theoretical points of view) incompleteness of presently available $\sigma_{o}(h \nu, T)$ spectra, the set of trap-specific parameters to be actually determined via least-mean-squares fittings reduced factually to the FC shift $D$, in combination with the first-order expansion coefficient $c_{1}$ (see Table I). Let us now comment on the results obtained for the individual deep levels under study.

For the thermal ionization energy $E^{T}$ of the $Z_{1 / 2}$ center in Eq. (2) we use a fixed value of $E_{A}-E_{\text {capture }}=0.55 \mathrm{eV}$, following from a measured DLTS electron emission energy value of $E_{A}=0.61 \mathrm{eV}$ and an intermediate value $E_{\text {capture }}=0.06 \mathrm{eV}$ for the thermal electron capture barrier. (Note that Hemmingsson et al. ${ }^{15}$ and Weidner et al. ${ }^{13}$ have reported $E_{\text {capture }}$ values of $0.065-0.080 \mathrm{eV}$ and $0.05 \pm 0.01 \mathrm{eV}$, respectively.) The least-mean-squares fit yielded a FC shift of $D$ $=1.32 \mathrm{eV}$, which corresponds to a classical optical ionization energy, $E^{O} \equiv E^{T}+D$, of $E^{O}=1.87 \mathrm{eV}$. The result of the numerical fit is shown by the solid curve in Fig. 3. In a previous paper we have quoted an $E^{O}$ value of about $1.5 \mathrm{eV}$ for this center. ${ }^{10}$ In that preceding paper, we adopted a conventional model for the electronic part of the optical cross section in combination with the high temperature limiting expression for the Franck-Condon factor, ${ }^{22,23}$ which is, however, factu- ally inapplicable to deep levels in wide band gap materials. ${ }^{20}$ In addition to this, the high-energy section of the present optical cross section curve (Fig. 3) is somewhat different from that shown in the preceding paper ${ }^{10}$ owing to further, careful corrections in the O-CTS data interpretation procedure. These changes in numerical analyses are the reason for the difference between previous and present $E^{O}$ values for this center. Concerning different $E^{O}$ values quoted in various papers for the $Z_{1 / 2}$ center, it may still be interesting to note that Weidner et al. have reported a significantly smaller $E^{O}$ value (lying in a range of $0.5 \mathrm{eV}<E^{O}<0.9 \mathrm{eV}$ ). ${ }^{13}$ In their measurements, the sample was continuously illuminated during the measurements. They suggested that $E^{O}$ should be located in that photon energy range where an appreciable optical excitation of electrons from the ground state began to be observable. This qualitative estimation was not underpinned, however, by any theoretical model and/or a detailed numerical analysis. We note that the actual optical absorption edge (for the $T=0$ limit) is coincident just with the thermal ionization energy, $E^{T} \equiv E^{O}-D$. Thus it is a matter of principle that the optical cross section is nonvanishing, $\sigma_{o}>0$, throughout the whole interval of photon between the thermal and the classical optical ionization energies, $E^{T}<h \nu<E^{O}$. Thus one has generally good chances to observe optical absorption processes already at photon energies well below $E^{O}$ (as shown in Fig. 3) when the illumination time, the flux, and the FC shift are sufficiently large. Thus, in view of an unusually large magnitude (of the order of $1.3 \mathrm{eV}$, Table I) for the FC shift of the $Z_{1 / 2}$ center, it is not surprising that an appreciable amount of trapped electrons can be excited from this level by light with energies of about, or even less than, $1 \mathrm{eV}$ (Fig. 3). Let us still present another argument in favor of our result, according to which $E^{O}$ for the $Z_{1 / 2}$ center must be much larger than $1 \mathrm{eV}$. Numerous analyses of optical cross section curves performed in the past, for a large variety of deep levels in various materials, have continually shown that $E^{O}$ is normally located somewhere in the vicinity of the turning point of the increasing part of the measured $\sigma_{o}>0$ curve. Now we see from Fig. 3 that the increasing part of this curve has a nearly linear section between about 1.5 and $1.9 \mathrm{eV}$ (where the shape obviously changes from concave to convex form). Although the actual position of the turning point cannot accurately be located, it is clear at least that it lies somewhere within this interval. Consequently, the classical optical ionization energy is likely to range also somewhere within this interval, i.e., $E^{O}=(1.7 \pm 0.2) \mathrm{eV}$ (in the roughest approximation). This is in at least qualitative accordance with the present estimate of $1.87 \mathrm{eV}$ (Table I), as well our preceding estimate of $1.5 \mathrm{eV}$, for $E^{O}$. In summary we can conclude that, despite all the still existing numerical uncertainties, the previously quoted magnitude of about $1.5 \mathrm{eV}$ (Ref. 10) can be looked upon as a lower boundary for $E^{O}$ of the $Z_{1 / 2}$ center. This implies that the estimate of $E^{O}<0.9 \mathrm{eV}$ by Weidner et al. ${ }^{13}$ is clearly inadequate.

In the case of the $\mathrm{EH}_{6 / 7}$ center, the electron emission energy value $E_{A}$ is $1.65 \mathrm{eV}$. In fact, $E_{A}=1.65 \mathrm{eV}$ was measured at around $600 \mathrm{~K}$ (Fig. 1 in Ref. 10), not at the O-CTS measurement temperature $(350 \mathrm{~K})$. The band gap shrinks by $0.1 \mathrm{eV}$ with an increase in temperature from 350 to $600 \mathrm{~K}$, 
and thus $E_{A}$ at $350 \mathrm{~K}$ would be different from $1.65 \mathrm{eV}$. However, we ignore this temperature effect because it is unknown whether the energy level of the $\mathrm{EH}_{6 / 7}$ center shifts with temperature accompanying the conduction band edge or the valence band edge. On the other hand, the electron capture cross section at the DLTS measurement temperature is very large ${ }^{17}$ which indicates a negligibly small magnitude of the thermal electron capture barrier $E_{\text {capture }}=0$. Accordingly, we identify the thermal ionization energy $E^{T}$, with $E_{A}=1.65 \mathrm{eV}$. The least-mean-squares fit by Eq. (2) yielded a FC shift of $D=0.30 \mathrm{eV}$, which corresponds to $E^{O}=1.95 \mathrm{eV}$. These $D$ and $E^{O}$ values include uncertainty due to the band gap variation with temperature, as described above, and also uncertainty due to the insufficient energy range of $\sigma_{o}$ data, as described later. The result of the numerical fit is shown by the solid curve in Fig. 3.

For the NB center (in analogy to the $\mathrm{EH}_{6 / 7}$ center) the electron capture cross section at the DLTS measurement temperature is also very large (comparable even to the limiting magnitude for infinitely high temperatures, Sec. III). Thus we identify again the thermal ionization energy $E^{T}$ with the measured DLTS electron emission energy value of $E_{A}$ $=0.8 \mathrm{eV}$. The least-mean-squares fit by Eq. (2) yielded a FC shift of $D=1.28 \mathrm{eV}$, which corresponds to $E^{O}=2.08 \mathrm{eV}$. The result of the numerical fit is shown by the solid curve in Fig. 3.

The state of affairs for the $\mathrm{RD}_{1 / 2}$ center is more complicated than for the other centers, since its DLTS signal consists of many peaks, as revealed by the short filling pulse DLTS measurements. Nevertheless, we tentatively perform the fitting in a way analogous to that for the other centers. Accordingly, we identify again $E^{T}$ with $E_{A}=1.01 \mathrm{eV}$. The least-mean-squares fit by Eq. (2) yielded then a FC shift of $D=1.24 \mathrm{eV}$, which corresponds to $E^{O}=2.25 \mathrm{eV}$. The result of the numerical fit is shown by the solid curve in Fig. 3 .

The entirety of parameter values (both of fixed and fitted ones) for the four deep levels under study are listed in Table I. As a somewhat surprising feature of this parameter collection one might perhaps consider, at first sight, the occurrence of unusually large values for the FC shift, $D>1 \mathrm{eV}$, for three of the four levels under study. This manifests an apparently general tendency for the FC shift of deep levels in $\mathrm{SiC}$ to be much larger (up to an order of magnitude) than in conventional semiconductor materials such as $\mathrm{Si}$ (Refs. 24-26) and GaAs. ${ }^{27}$ On the other hand, large FC shifts (of order $10^{0} \mathrm{eV}$ ) have been found already years ago (e.g., for the $\mathrm{O}$ donor level in GaP (Ref. 28) and numerous $D X$ donor levels in $\left.\mathrm{Al}_{x} \mathrm{Ga}_{1-x} \mathrm{As}\right)^{29-32}$ as well as in a series of other ternary III-V compounds. ${ }^{32}$ More recently, a FC shift of order $1 \mathrm{eV}$ has also been found for a deep level in $\mathrm{GaN}^{20}$ Concerning the presently studied deep levels in $4 H$-SiC, we have found here, particularly for the $Z_{1 / 2}$ and the NB center, that the FC shift is significantly larger than the thermal ionization energy, $D$ $>E^{T}$ (corresponding to the regime of large lattice relaxation). ${ }^{8,20,28,29,32}$ A duly strong coupling with the crystal lattice could be expected in particular for the the $Z_{1 / 2}$ center, since this has been reported to be a negative- $U$ center. ${ }^{13,15}$ Such negative- $U$ centers generally involve large lattice relaxation energies, since this is a prerequisite for them to be more stable in the two electron capture state than in the one electron capture state. ${ }^{8,32}$ Thus it is quite plausible that the negative- $U Z_{1 / 2}$ center is associated with a large FC shift $(D>1 \mathrm{eV})$.

At the same time we must be conscious of the fact that the presently quoted parameter values can by no means be looked upon as definitive ones. Particularly, the estimated optical ionization energies $E^{O}$, which correspond to the sums of the (fixed) thermal ionization energies $E^{T}$ and the associated (fitted) FC shifts, $E^{O}$, are strongly correlated ${ }^{21}$ with the analytical model actually chosen for the electronic part of the optical cross sections. Fortunately, even with respect to the estimated optical ionization energies $E^{O}$, the existing uncertainties are not excessively large. Referring once more to the general property (mentioned above) that $E^{O}$ is normally located near the turning point of the increasing part (and thus somewhere within the approximately linear section) of the optical cross section curve, we can readily estimate $E^{O}$ from Fig. 3 for the $\mathrm{NB}$ center and the $\mathrm{RD}_{1 / 2}$ center to be about $(1.8 \pm 0.2)$ and $(2.0 \pm 0.2) \mathrm{eV}$, respectively. In both cases, the $E^{O}$ values obtained above by numerical fittings are close to (slightly higher than) the upper boundaries of the intervals estimated simply by viewing the experimental curves. In summary we can assess that, for the $Z_{1 / 2}$, the $\mathrm{NB}$, and the $\mathrm{RD}_{1 / 2}$ center, the maximum uncertainties of optical ionization energies $E^{O}$ are clearly limited to an order of $\pm 0.3 \mathrm{eV}$ (with respect to the centers of gravity of about 1.7, 1.8, and $2.0 \mathrm{eV}$, respectively, of the $E^{O}$ intervals estimated above). However, in contrast to these centers, the $\sigma_{o}$ for the $\mathrm{EH}_{6 / 7}$ center (Fig. 3) is monotonically increasing, and its shape remains concave, even up to the highest photon energy $(2.38 \mathrm{eV})$ achieved in the experiment. No turning point, or no associated nearly linear section, can be detected from this curve. This means that the $E^{O}$ values of about $1.95 \mathrm{eV}$, resulting from our numerical fit (Table I), is largely uncertain. This value can be looked upon, at best, as a lower boundary for $E^{O}$. At the same time it appears from Fig. 3 that the actual $E^{O}$ might be even significantly higher than $2.4 \mathrm{eV}$. Thus the parameter uncertainty for the $\mathrm{EH}_{6 / 7}$ center is markedly larger than for the other centers.

On the basis of known $D$ and $E^{T}$ values it is possible, in principle, to precalculate the temperature dependences (as relative values) of the associated thermal electron capture cross sections $\sigma(T)$ that are due to the NMP capture mechanism. ${ }^{5,21}$ [Note that absolute $\sigma(T)$ can be measured by short filling pulse DLTS measurements, but only at the DLTS measurement temperature.] Within the frame of the effective phonon energy approximation, ${ }^{21}$ we can represent the $\sigma(T)$ dependence roughly by Eq. (11) in Ref. 21. Figure 9 shows the calculated temperature dependences of the NMP capture cross sections for the $\mathrm{NB}$ and $Z_{1 / 2}$ centers. (Note that analogous curves for $\mathrm{EH}_{6 / 7}$ and $\mathrm{RD}_{1 / 2}$ centers are omitted here in view of their larger parameter uncertainties, as discussed above). In view of the present lack of knowledge of the actual, trap-specific magnitudes of the high temperature limits, $\sigma_{\infty}$, we have presented these temperature dependences in terms of the ratios $\sigma(T) / \sigma_{\infty}$ (implying a normalization of the high temperature limits to unity). The $\sigma(T)$ dependence for the NB center is seen to be very weak. This is consistent with 


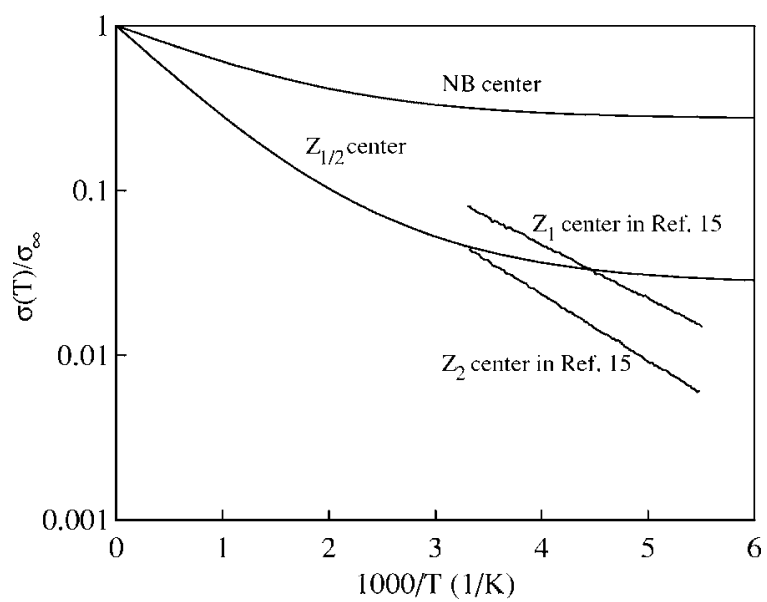

FIG. 9. Temperature dependences of the thermal electron nonradiative multiphonon capture cross sections for the $\mathrm{NB}$ and $Z_{1 / 2}$ centers calculated from Eq. (11) in Ref. 21. Included are experimental capture cross-section values for the $Z_{1}$ and $Z_{2}$ centers reported in Ref. 15 .

the results of our DLTS measurements, from which an appreciable temperature dependence of the capture cross section was not detectable. The magnitude of $\sigma(T)$ should thus be nearly equal to $\sigma_{\infty}$ (i.e., of an order of $10^{-15}-10^{-14} \mathrm{~cm}^{2}$, at any temperature). The calculated $\sigma(T)$ for the $Z_{1 / 2}$ center shows a somewhat stronger decrease, with decreasing temperature, and becomes temperature independent at low temperatures. Hemmingsson et al. ${ }^{15}$ have reported experimental data of $\sigma(T)$, which have been separately measured for the $Z_{1}$ and $Z_{2}$ centers (as shown in Fig. 9). As mentioned above, the $Z_{1 / 2}$ center is a negative- $U$ center and thus involves two electron capture processes (the first one into the $Z_{1 / 2}^{+}$and the second one into the $Z_{1 / 2}^{0}$ state). Hemmingsson et al. ${ }^{15}$ have reported that the electron capture process into the $Z_{1 / 2}^{+}$state is so fast that it is practically impossible to observe it experimentally. Their experimental data for $\sigma(T)$ correspond thus to the electron capture process into the $Z_{1 / 2}^{0}$ state. Our calculation of the $\sigma(T)$ dependence for the $Z_{1 / 2}$ center is based on results of our DLTS and O-CTS measurements of thermal and optical electron excitation processes, which proceed again in the form of two electron excitation processes. The first one from the $Z_{1 / 2}^{0}$ state is fast (because of its smaller activation energy) in comparison with the second one from the $Z_{1 / 2}^{-}$state. Thus we monitored in our O-CTS and DLTS measurements essentially the processes of excitation of the second electron from the $Z_{1 / 2}$ state. Consequently, the levelspecific parameter values, including the calculated $\sigma(T)$ dependence (Fig. 9), refer to the electron capture into the $Z_{1 / 2}^{0}$ state, on the one hand, and the electron emission from the $Z_{1 / 2}^{-}$state, on the other hand. Thus the calculated and experimental $\sigma(T)$ curves in Fig. 9 refer to the same process, namely the electron capture process into the $Z_{1 / 2}^{0}$ state. However, as we see from the comparison in Fig. 9, the calculated $\sigma(T)$ curve shows a rather weak dependence below $300 \mathrm{~K}$, whereas the experimental curves show even in this region a relatively strong exponential dependence. This discrepancy might be due to the circumstance that we have lumped here the $Z_{1}$ and $Z_{2}$ centers into a single center, and/or the chosen value of $80 \mathrm{meV}$ for the effective phonon energy $\varepsilon$ might have been too high in this case. With decreasing $\varepsilon$, the range of exponential (Arrhenius type) temperature dependence would further expand towards lower temperatures. Thus a reduction of $\varepsilon$ would markedly reduce the existing discrepancy between theoretically estimated and experimentally measured $\sigma(T)$ dependences for the $Z_{1 / 2}$ center.

\section{CONCLUSION}

Deep levels in two $4 \mathrm{H}$-SiC epilayers grown by cold wall chemical vapor deposition were characterized by the deep level transient spectroscopy (DLTS) and the opticalcapacitance-transient spectroscopy (O-CTS). Observed DLTS peaks corresponded to the previously reported $Z_{1 / 2}$, $\mathrm{EH}_{6 / 7}$, and $\mathrm{RD}_{1 / 2}$ centers, and a newly observed NB center. By O-CTS measurements, optical cross section spectra for these centers were obtained, and the corresponding FranckCondon shifts (or optical ionization energies) were estimated via least-mean-squares fittings using a sufficiently general theoretical model. The level-specific Franck-Condon shifts, which are given by the differences between optical and thermal ionization energies, were found to be relatively large for these centers in comparison with those known from earlier papers for deep levels in conventional semiconductor materials. On the basis of the estimated Franck-Condon shifts and thermal ionization energies, we have calculated for $Z_{1 / 2}$ and NB centers the respective temperature dependences of thermal electron nonradiative multiphonon capture cross sections.

\section{ACKNOWLEDGMENTS}

We would like to thank Dr. G. Pensl, Dr. M. Weidner, and Dr. S. Reshanov, Universität Erlangen-Nürnberg, Germany, for their fruitful discussion.

${ }^{1}$ H. McD. Hobgood et al., Mater. Sci. Forum 457-460, 3 (2004).

${ }^{2}$ K. Danno, K. Hashimoto, H. Saitoh, T. Kimoto, and H. Matsunami, Jpn. J. Appl. Phys., Part 2 43, L969 (2004).

${ }^{3}$ T. Tawara, H. Tsuchida, S. Izumi, I. Kamata, and K. Izumi, Mater. Sci. Forum 457-460, 565 (2004).

${ }^{4}$ J. Zhang, L. Storasta, J. P. Bergman, N. T. Son, and E. Janzén, J. Appl. Phys. 93, 4708 (2003).

${ }^{5}$ R. Pässler, Phys. Status Solidi B 68, 69 (1975); 76, 647 (1976).

${ }^{6}$ C. H. Henry and D. V. Lang, Phys. Rev. B 15, 989 (1977).

${ }^{7}$ Y. Nakakura, M. Kato, M. Ichimura, E. Arai, and Y. Tokuda, Mater. Res. Soc. Symp. Proc. 719, 167 (2002).

${ }^{8}$ Y. Nakakura, M. Kato, M. Ichimura, E. Arai, Y. Tokuda, and S. Nishino, J. Appl. Phys. 94, 3233 (2003).

${ }^{9}$ M. Kato, M. Ichimura, E. Arai, and Y. Tokuda, Defect Diffus. Forum 218-220, 1 (2003).

${ }^{10}$ M. Kato, S. Tanaka, M. Ichimura, E. Arai, S. Nakamura, and T. Kimoto, Mater. Sci. Forum 483-485, 381 (2005).

${ }^{11}$ S. A. Reshanov, K. Schneider, R. Helbig, G. Pensl, H. Nagasawa, and A. Schöner, Mater. Sci. Forum 457-460, 513 (2004).

${ }^{12}$ S. A. Reshanov and V. P. Rastegaev, Diamond Relat. Mater. 10, 2035 (2001).

${ }^{13}$ M. Weidner, G. Pensl, H. Nagasawa, A. Schöner, and T. Ohshima, Mater. Sci. Forum 457-460, 485 (2004).

${ }^{14}$ S. Nakamura, T. Kimoto, and H. Matsunami, Jpn. J. Appl. Phys., Part 1 41, 2987 (2002).

${ }^{15}$ C. G. Hemmingsson, N. T. Son, A. Ellison, J. Zhang, and E. Janzén, Phys. Rev. B 58, R10119 (1998).

${ }^{16}$ T. Dalibor, G. Pensl, H. Matsunami, T. Kimoto, W. J. Choyke, A. Schöner, and N. Nordell, Phys. Status Solidi A 162, 199 (1997).

${ }^{17}$ C. G. Hemmingsson, N. T. Son, O. Kordina, J. P. Bergman, E. Janzén, J. 
L. Lindström, S. Savage, and N. Nordell, J. Appl. Phys. 81, 6155 (1997).

${ }^{18}$ R. Pässler, Phys. Status Solidi B 170, 219 (1992).

${ }^{19}$ R. Pässler, Phys. Status Solidi B 179, 133 (1993).

${ }^{20}$ R. Pässler, J. Appl. Phys. 96, 715 (2004).

${ }^{21}$ R. Pässler, J. Appl. Phys. 97, 113533 (2005).

${ }^{22}$ A. Chantre, G. Vincent, and D. Bois, Phys. Rev. B 23, 5335 (1981).

${ }^{23}$ M. Jaros, Phys. Rev. B 16, 3694 (1977).

${ }^{24}$ R. Pässler, H. Pettersson, H. G. Grimmeiss, and K. Schmalz, Phys. Rev. B 55, 4312 (1997)

${ }^{25}$ H. Pettersson, R. Pässler, F. Blaschta, and H. G. Grimmeiss, J. Appl. Phys. 80, 5312 (1996).
${ }^{26}$ R. Pässler, H. Pettersson, and H. G. Grimmeiss, Semicond. Sci. Technol. 11, 1388 (1996).

${ }^{27}$ G. F. Neumark and K. Kosai, Semicond. Semimetals 19, 1 (1982).

${ }^{28}$ H. Kukimoto, C. H. Henry, and F. R. Merritt, Phys. Rev. B 7, 2486 (1973).

${ }^{29}$ D. V. Lang and R. A. Logan, Phys. Rev. Lett. 39, 635 (1977).

${ }^{30}$ D. V. Lang, R. A. Logan, and M. Jaros, Phys. Rev. B 19, 1015 (1979).

${ }^{31}$ R. Legros, P. M. Mooney, and S. L. Wright, Phys. Rev. B 35, 7505 (1997).

${ }^{32}$ P. M. Mooney, J. Appl. Phys. 67, R1 (1990). 